\title{
THE DEVELOPMENT OF CHILDREN'S KNOWLEDGE: THE SKY, THE EARTH AND THE SUN IN CHILDREN'S EXPLANATIONS
}

\section{Eve Kikas}

\begin{abstract}
Our knowledge about the world is mediated by sense organs, material and mental mediators, which convey information that may appear conflicting. This is why both children and adults experience difficulties in integrating and conceptualising the information. The article examines the development of children's knowledge from initial beliefs and knowledge to synthetic and scientific knowledge. Empirical studies investigating the knowledge of Estonian children about planet Earth, the possibility of living on earth and reasons for seasonal changes are described. Examples from interviews with children illustrate the complexity of constructing and conceptualising knowledge.
\end{abstract}

Keywords: initial beliefs, knowledge about astronomy, synthetic knowledge, development of knowledge, mediated knowledge.

"The children would remember for the rest of their lives the august solemnity with which their father, devastated by his prolonged vigil and by the wrath of his imagination, revealed his discovery to them:

"The earth is round, like an orange." Úrsula lost her patience. "If you have to go crazy, please go crazy all by yourself." she shouted. "But don't try to put your gypsy ideas into the heads of the children."

(Gabriel Garcia Marquez One Hundred Years of Solitude)

People perceive the world through mediation. The knowledge of animals, children and adults is mediated by sense organs. People also use other mediators ("tools"), which enable to perceive the world more accurately, in greater detail and in a more complex manner. The tools may be either material (e.g. a spyglass or a telescope) or mental (e.g. language, models, theories of mathematics and phys- 
ics) (Kikas 2003; Nelson 2003; Toomela 2003; Vygotsky 1931/1983; Wertsch 1998). The mediated perception of the world is represented in Figure 1. Certain material mediators directly expand the observed phenomena, some devices and theories enable us to look even further. Theories in particular render the space, galaxies, black holes perceptible (or cognisable) for us. New mediators - both mental and material - are constructed on the basis of old ones. In addition, the majority of our knowledge is socially created, which means that the creation of previous generations has been accumulated in material and mental mediators. Also, children need to acquire the knowledge not only through directly experiencing the world but also from other people who help them conceptualise symbols in a culturally acceptable form (Nelson 2003; Tomassello 2000).

Modern societies have more powerful devices and more complicated theories than societies in the past; the devices possessed by some societies are better than those of others. The majority of people have no access to powerful mechanisms, neither are they familiar with modern theories. The children's set of mediators or "tools" is even more limited. Therefore it is quite understandable that the knowledge of children, adults and scholars studying at different periods differ both in quantity (scholars have more knowledge in greater detail about the world than children) and in quality (their reasoning differs) (on scholarly theories see Kuhn 1962; on children see Vosniadou 1994a).

Also, it is a common knowledge that conceptualisation and explanation of space and its regularities has differed throughout history (on the Estonian material see Kuperjanov 2003).

Apart from its primary role in perceiving the world, (symbolic) mediation is also important in making one's knowledge known to others (see Figure 1). One's knowledge is generally expressed by means of words. The meaning of words has been and should be established on a common basis to avoid misinterpretation and misunderstanding. Specialised terminology is coherent and uniformly understood among the specialists of specific areas of research, but the meaning of the terms may differ in general context and in the lexica of other fields of speciality. Non-specialists may therefore interpret scientific explanations in their own way (i.e. hold misconceptions) (e.g. 


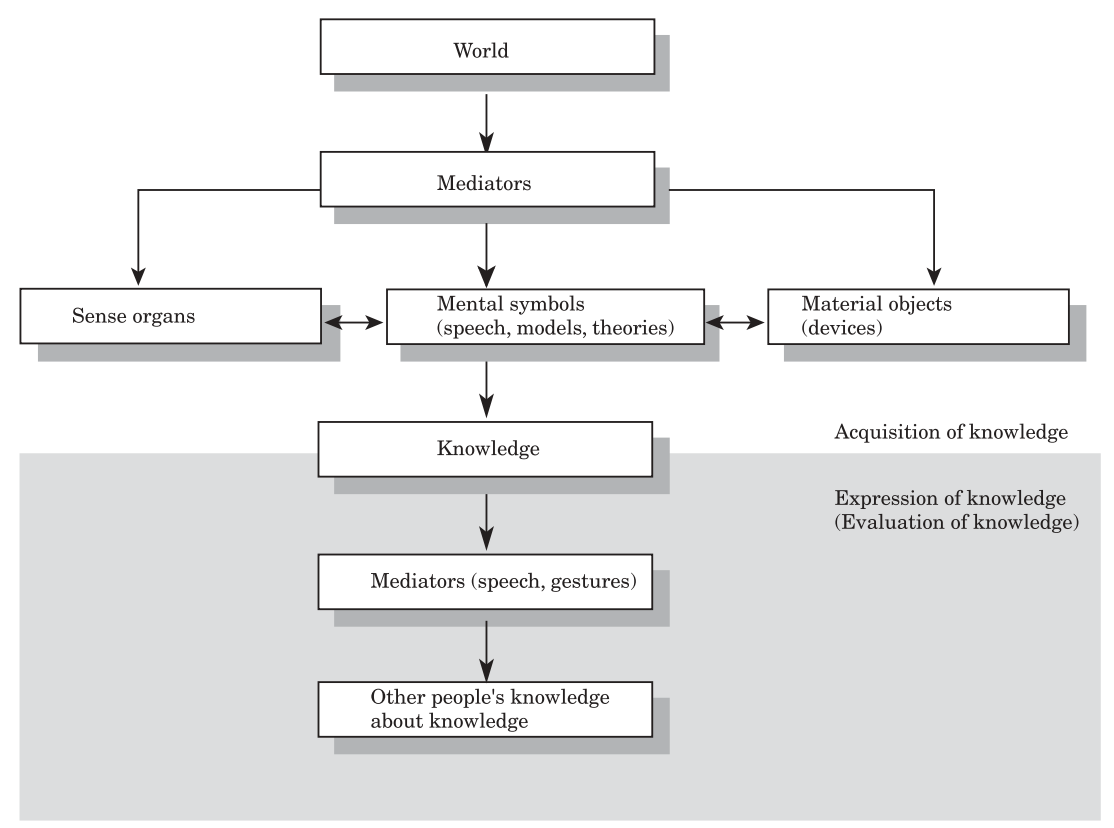

Figure 1. Mediation during acquiring and expressing knowledge

Brewer \& Chinn et al 2000; Driver \& Squirer et al. 1995). Understanding speech and making oneself understandable to others has proven particularly difficult for children who are in the middle of the process of learning words and their meanings and whose cognitive abilities are limited.

In the present article I will describe the development of children's knowledge and point out the reasons behind acquiring new information and editing the already existing knowledge. Provided examples are borrowed from the area of astronomy, demonstrating the problems that children experience in conceptualising the earth as a planet, and understanding the earth's gravitational force and seasonal changes. 


\section{INITIAL BELIEFS}

The knowledge of infants and young children is mediated via sense organs: the child's attention is focused directly on objects (see Fig. 1). Around the age of 9 months infants start participating in activities which demand the joint attention of adults (Tomassello 2000). From this moment on the child can be taught the meanings of words, for which it has to look at the objects that adults point out; from this point the child learns to mediate the world through mental symbols. Parents and other adults help children in perceiving the world by showing them objects or images and associating these with corresponding words: as a result, the child will learn to understand speech. A one-year-old begins to utter the first words but also to point to objects, demanding new words; in this process the common knowledge of a child and an adult is developed (Tomassello 2000). Initially, infants imitate the speech of adults like parrots, and only later they start constructing sentences independently and more creatively.

Since infants and toddlers do not know how to mediate their knowledge to others - as they do not speak -, the extent of their knowledge is relatively difficult to study. Up to the point where children's knowledge was investigated only in terms of word mediation, children were thought of being egocentric and their knowledge nonexistent. A few decades ago, however, when scholars started to 'measure' infants' knowledge on the basis of the eye movements (tasks, in which children are less restricted compared to speech), it was found that children possess considerably more advanced presuppositions of the world. It was discovered that infants display special conceptual and perceptual structures (beliefs), which enable them to integrate and interpret different pieces of the world (Baillargeon 1995; Spelke 1991). These structures enable the child to select from an immense amount of information what they require for successful and safe activity. For example, infants understand that a solid body cannot pass through another solid object, that two solid bodies cannot be located in the same spatial point (Spelke 1991).

Relying on the works by Spelke, Baillargeon and other authors, Vosniadou (1994a) described two important beliefs defining and guiding the children's perception of the earth, the sky and space: (i) the space is arranged in a way that the up/down direction is firmly 
established and functions transversely in relation to the flat ground; (ii) objects move downward in the absence of support.

\section{INITIAL KNOWLEDGE}

Children develop in the world of meanings shared with adults; the acquisition of the meanings of words is done via language and other symbols (Nelson 2003). The words of three-year-old children signify specific objects, actions, phenomena associated with the nearest surroundings. At this age children still learn the meanings of words by pointing to an object or directly asking 'What is it?' But they will also begin to actively find explanations about the world by asking questions of 'why?' and 'how?' (Wellman \& Hickling et al. 1997). Callanan and Oakes (1992) have shown that these questions are mostly asked during everyday activities shared by an adult and a child. Children's questions do not consist of a single word but are expressed in full sentences (e.g. 'Where does the rain come from?'); adults provide thorough explanations (e.g. 'First raindrops gather in a cloud, and when the cloud is full of them, the raindrops will fall down.'). Even though everything experienced through perception is of considerable importance at this age, knowledge is increasingly becoming mediated through mental symbols.

Children experience problems in expressing their thoughts and passing on their knowledge to others, which further complicates the study of the actual extent of their knowledge. Children's explanations (the level, complexity, integration) are affected by situation, interviewers, asked questions (Siegler 1996). In everyday situations and in the presence of familiar people, children display more advanced knowledge than when interviewed by unfamiliar experimenters. Causal explanations have been found when children are being tape-recorded at play (both with peers and adults)(Callanan \& Oakes 1992; Wellman \& Hickling et al. 1997), but children still provide egocentric explanations to direct questions (e.g. 'It is raining because then we can play in puddles'). Children exhibit more advanced knowledge if models and other material aids are being used during interviews (Schoultz \& Säljö et al. 2001). Questions asked by adults on more than one occasion (about the same matter, but at a different angle, at least from the adult's point of view) confuse children, and the latter may start contriving new variants for 
the response. Children are unable to provide answers to general and abstract questions, but specific questions often tend to be leading and force the children to think in a certain way (Nobes \& Moore et al 2003).

\section{Initial knowledge about the earth and the sky}

Currently there is no common agreement on the initial astronomical knowledge of pre-schoolers (cf. Kikas 2003; Nobes \& Moore et al. 2003; Vosniadou \& Brewer 1992). Some studies have found that children's explanations are still egocentric and relatively fragmentary. For example, the function of phenomena is explained through their usefulness to the child and other people ('The sun is shining so that it would warm us.' 'The night exists for us to be able to sleep'). Other studies have indicated the initial integrated knowledge of children, which are often referred to as common theories (on other areas of study see e.g. Gopnik \& Meltzoff 1998).

Vosniadou and her colleagues have described integrated astronomical knowledge and models. They have conducted investigations in several countries and discovered original models of the earth and corresponding explanations about the day/night cycle and the change of seasons, which are relatively similar in different cultures (Diakodou \& Vosniadou et al. 1997; Samarapungavan \& Vosniadou et al. 1996; Vosniadou \& Brewer 1992, 1994). Vosniadou proceeds from these results concluding that a child constructs initial models by itself, relying on initial beliefs (the space is arranged in a way that up/down is firmly established and functions transversely in relation to the flat ground, and objects move downward in the absence of support) and observations (the sun is up in the sky in daytime, but the moon and stars at nights). In addition, Vosniadou and Brewer (1992) claim that since children have no perception of infinite objects, they believe that the earth is finite and has an edge. Since the surface of the earth appears flat, objects fall down and the earth must have an edge, children construct the initial disc-shaped or rectangular model of the earth (Diakodoy \& Vosniadou et al. 1997; Vosniadou 1994a, 1994b; Vosniadou \& Brewer 1992, 1994). The same beliefs were known in Estonia and elsewhere in the past (Kuperjanov 2003). Vosniadou and her colleagues interviewed children and asked them to draw pictures and explain their drawings. A major weakness of their study, however, is that children are di- 
rectly asked about the edge of the earth ('If you walked and walked in a straight line, where would you end up? Would you ever reach the edge of the earth? Can people fall off the end/ edge of the earth?'). As mentioned above, children can be easily manipulated by adults and leading questions often result in affirmative answers, even if the children have not previously thought about the issue.

In Estonia, similar questions have been used while interviewing children, though the word 'edge' has not been directly used. For the purpose of a study 144 pre-schoolers (69 boys and 75 girls) were interviewed for four times with a one-year interval. At the time of the first interview the children were 3 years old and were only beginning to construct their first sentences and make their first drawings. The children were asked the following questions about the earth:

1. What is the shape of the earth, where people live on? If the answer was round, two models were shown towards the end of the interview - a paper disc and a table tennis ball - and they were asked: Which round object is the earth - this or that?

2. If you walked and walked in a straight line, where would you end up? If you walked even further, where would you end up?

3. Can you fall down from the earth? If the child replied affirmatively, the next question was: Where?

As was expected, fewer children referred to the edge of the earth than those who were directly asked about it. Furthermore, the third question is relevant only when the child believes that the earth has an edge. Therefore many children reinterpreted the question as ' $\mathrm{Can}$ you fall off?'. Table 1 indicates the number of children relative to years, who gave no reply, who provided the correct answer and who made independent reference to the edge of the earth (those who pointed to the disc-shaped model of the earth, replied that walking takes one to the edge of the earth and one can fall down over the edge). As indicated, several 3-year-olds already replied that people can fall over the edge of the earth. Children tend to select the disc model at the age of 5 and 6 , rather than at the age of 3 or 4 . With the advancement of age, the number of children who believe that by walking directly one can end up at the edge of the earth increases. 


\begin{tabular}{|l|r|r|r|r|r|r|r|r|r|r|r|r|}
\hline & \multicolumn{3}{|c|}{ 3-year-olds } & \multicolumn{4}{|c|}{ 4-year-olds } & \multicolumn{3}{|c|}{ 5-year-olds } & \multicolumn{3}{|c|}{-year-olds } \\
\hline Question & D & E & C & D & E & C & D & E & C & D & E & C \\
\hline $\begin{array}{l}\text { The shape } \\
\text { of the }\end{array}$ & 139 & 1 & 3 & 102 & 19 & 21 & 66 & 33 & 39 & 27 & 23 & 90 \\
earth & & & & & & & & & & & & \\
Walking & 76 & 0 & 0 & 50 & 7 & 4 & 37 & 16 & 0 & 33 & 41 & 14 \\
Falling & 10 & 39 & 0 & 3 & 43 & 4 & 1 & 32 & 1 & 2 & 30 & 8 \\
\hline
\end{tabular}

Table 1. Distribution of children's responses.

Note: Some (the sc. descriptive) answers have not been included in the Table, which explains the difference in the number of respondents of different ages and questions. $\boldsymbol{D}$ don't know, $\boldsymbol{E}$ - edge, $\boldsymbol{C}$ - correct.

The responses reveal that some preschoolers conceptualise the earth as a finite object with an edge. Our study, however, revealed that responses given to different questions are inconsistent with each other. Thus we could not conclude that the majority of children possess fixed models of the earth (e.g. disc, rectangle). Some children, for example, selected a sphere as a model of the earth, but were still convinced that it is possible to fall over the edge of the earth or that by walking directly one may end up at the edge of the earth.

\section{SYNTHETIC KNOWLEDGE}

Even though specific experience and directly perceived close surroundings play a major role in children's life in preschool age and in elementary school, an increasing amount of information is being mediated through symbols, mainly through language. As mentioned in the previous chapter, adults explain children various phenomena of the world by simplifying scientific theories, though still in an abstract way (Callanan \& Oakes 1992). In school, information about new phenomena, which cannot be directly observed, is acquired. Children no longer use words of a visible referent, but words which are used to explain other words, i.e. the world of symbols is distinguished from the world of objects (Vygotsky \& Luria 1997). Wertsch (1985: 33) describes the process as decontextualisation of cultural mediators: the process whereby the meaning of signs becomes less and less dependent on the unique spatiotemporal context in which they are used. This language usage must be learned and the learn- 
ing mediated through signs takes time, at least when the learned material is to be understood and integrated into the framework of stored knowledge.

Preschoolers synthesise (construct) their knowledge of the world through the mediation of sense organs, language, stored knowledge, or two sources - observation of the world and explanations of adults (Kikas 2003; Nelson 2003; Vosniadou 1994a; Vygotsky 1997). Some authors argue that the selection and interpretation of information is guided by initial beliefs (Vosniadou 1994a; Vosniadou \& Brewer 1992).

Listening and interpreting adult speech is of great significance in constructing new explanations, because adults provide the vocabulary for children by answering their questions, by helping to structure information (Nelson 2003). Children do not take over the adults' explanations directly; knowledge cannot be "poured" in someone's head, but children have to interpret the explanations according to the stored knowledge and mental structure and, by doing that, integrate the knowledge into the structural framework. This process of interiorisation passes through multiple stages and requires time (Vygotsky 1997).

In the first stage a child hears a word or an explanation by an adult. Knowledge is acquired during a shared activity; it is a shared social knowledge. The child starts using the word and explanation in a way which appears similar to that of the adult, imitating that, though in reality the meaning of concepts used by the child may be quite different. Vygotsky (1997) refers to such concepts as pseudo-concepts, which adults generally regard as an indication that a child has acquired a new concept (knowledge). During this stage the application of new knowledge is limited strictly to situations analogous to that of acquisition. This knowledge is literally "poured" in the child's head; it is mechanically acquired (see the above about infants).

During the next stage, which is more time-consuming, meaning has to be attached to the knowledge and the knowledge integrated within the stored knowledge. If the stage is passed too quickly (e.g. in school where the rate of teaching is too high, the number of pupils in a class is large and the instruction is directed to the average 
level of the class) the knowledge may remain verbal (and is called verbalism or inert knowledge)(Chinn \& Brewer 2000; Vosniadou 1994a; Vygotsky 1997). Also, distorted knowledge (sometimes called naïve, misconceived or common knowledge) may develop (Driver \& Squirer et al. 1995; Glynn \& Duit 199). New mediators allow us to explain the world in a more integrated way, encompassing a wider range of phenomena, enabling new predictions (cf. theories and laws by Newton and Einstein) (Chinn \& Brewer 2000; Kuhn 1962). At the same time they limit understanding, as more prior knowledge and skills are required to understand them. Modern theories of physics and astronomy remain incomprehensible even to many adults, even though they may get excited about popular scientific explanations. Schoolchildren reinterpret information (distorting knowledge) by adapting it to their previous experience (see Chinn \& Brewer 2000). These explanations form a compromise between common knowledge and new information; they provide the learner with a sense of understanding, which they desperately need (Brewer \& Chinn et al. 2000). At best, schoolchildren acquire scientifically accurate knowledge.

The knowledge of schoolchildren and adults is also difficult to study, although reasons for it differ from those in the study of infants. Older children have acquired words, sentences, models for solving tasks, etc.; they impart their knowledge by imitating what they have learned at school. What makes the study difficult is the understanding whether the knowledge of pupils are verbalisms, distorted or accurately scientific knowledge. In these studies, differently formulated questions and tasks are used in order to obtain information through the mediation of means the use of which has not been taught. Consequently, the mediators up and below in Figure 1 must be different (see Kikas 1998a, 1998b;Vosniadou 1994a).

In the following I will illustrate the synthesising of knowledge and problems in understanding the narrower areas of conceptualising the earth with examples about the shape of the earth, the possibility of living on the earth and the change of seasons. Specific examples have been borrowed from studies conducted in Estonia (Hannust \& Kikas 2002; Kikas 1998a, 1998b, 2000; Kikas \& Hannust et al. 2002). The names of children used in the examples have been changed. 


\section{Synthetic knowledge: the shape of the earth and the possibility of living on the earth}

Children have no problems acquiring the fact that the earth is round, although the fact often remains a verbalism with no meaning attached to it. Understanding how it is possible to live in the different areas of the earth and not fall down (the concept 'down' is absolute here, as it is an initial belief, which up to this point has functioned as auxiliary, but has become to hinder development) is much more complicating (Vosniadou 1994b; Vosniadou \& Brewer 1992, 1994). Vosniadou and her colleagues have shown that children synthesise various models of the earth, which allow to join the visible (flat surface) to the audible (the earth is round) (Diakodoy \& Vosniadou et al 1997; Vosniadou 1994a, 1994b; Vosniadou \& Brewer 1992, 1994). Some children believe in the existence of two earths: one which we live on and the other which is a planet in the sky. Others believe that people live on a flat surface in the bottom of a hollow spherical earth, where the sky is located above the earth forming the upper part of the sphere. Yet other children believe that people live on the upper side of a flattened sphere. Such models enable to maintain a conception of the fixed up/downward direction.

Figure 2 presents different models of the earth as drawn by Estonian children. The models typically represent a round earth, though people and clouds have been delineated in the absolute up/down direction. Some children, however, have depicted people as living in the lower side inside a hollow sphere. Children also draw people as living on the upper hemisphere. The images of the models do not indicate whether the models reflect the child's difficulties in understanding the world or the child's difficulties in expressing the models (see Figure 1). Children's drawings reflect what they know and believe, though on the other hand they may display inadequate drawing skills (Blades \& Spencer 1994). Smaller children often draw according to previously acquired schemes and experience difficulties in changing these schemes when given a different task (KarmiloffSmith 1992). Children most often draw people at the bottom of the sheet and the sky at the top, and may follow this scheme while drawing the earth.

Scholars agree in that children experience great difficulties in understanding the modern scientific model of the spherical earth. Some 


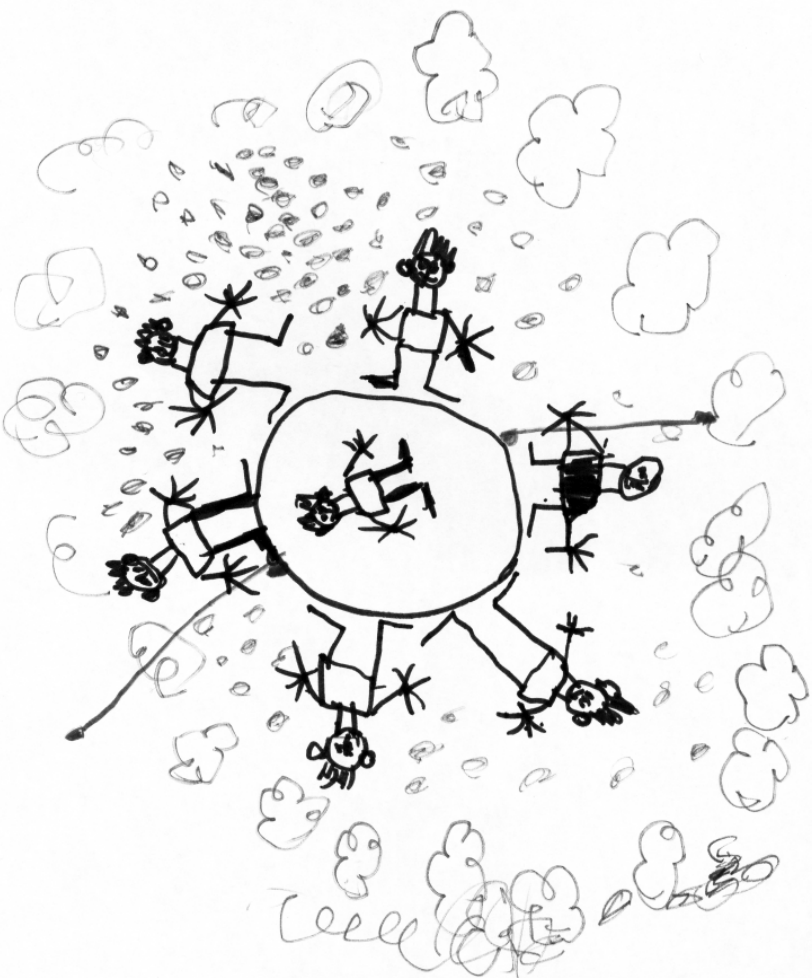

Figure $2 a$.
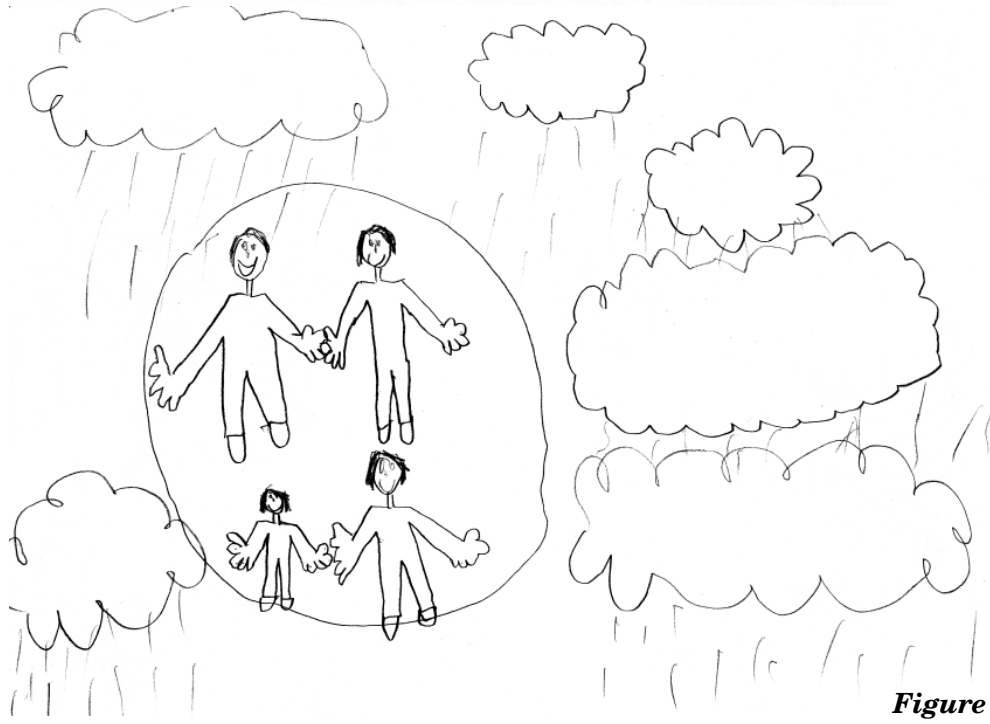

Figure $2 b$. 


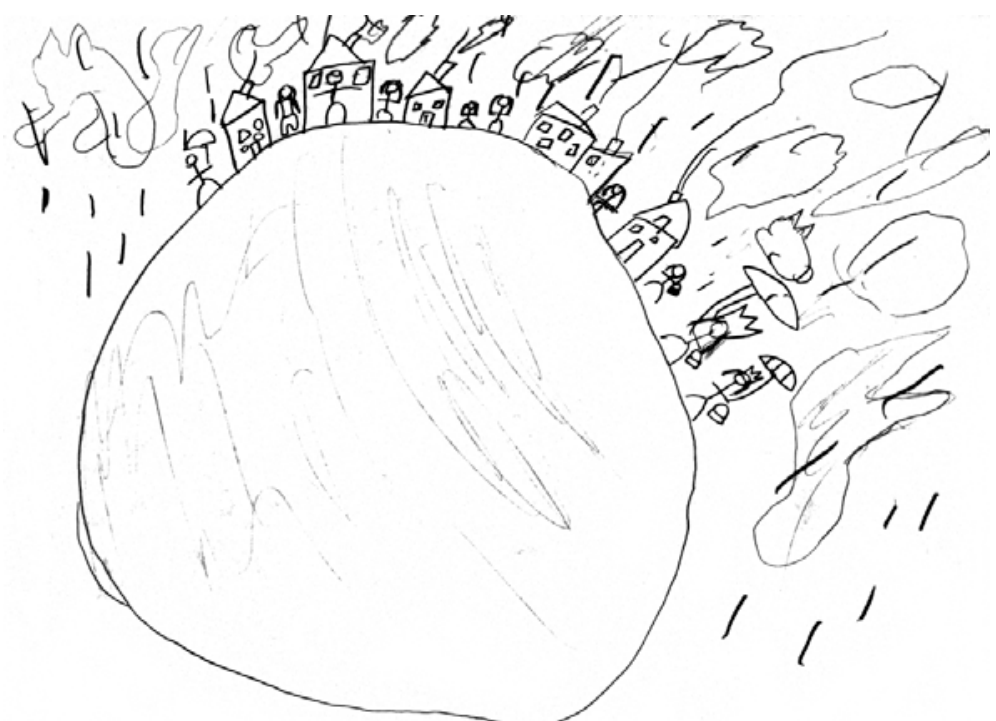

Figure 2c.

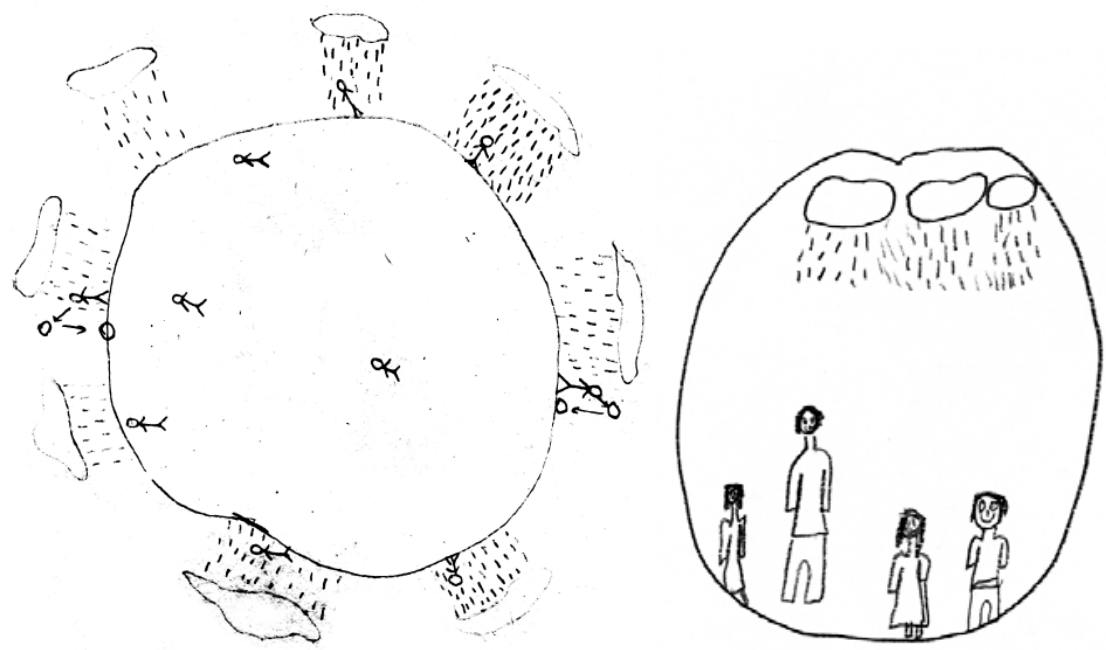

Figures 2d-e.

Figure 2a-e. Depiction of the earth, people, clouds and rain in children's drawings. 
children have not acquired the knowledge by the end of the elementary school; some studies have indicated that even adults foster various misconceptions (Vosniadou 1994a). Instructional experiments have been conducted in Estonia, during which initial knowledge connected to the shape of the earth and gravity have been taught to children ranging in the age from 5 to 7 (Hannust \& Kikas 2002; Kikas \& Hannust et al. 2002). In the course of these experiments the total of a hundred children ranging in the age 5-7 has been educated. Hele Kanter carried out individual tutoring, Triin Hannust instructed children in small groups by using models. Children's knowledge was evaluated by interviewing and drawings. None of the interviewed children had prior knowledge of the scientific model of the earth: some believed the earth to be flat or could say nothing about its shape; others possessed the factual knowledge that the earth is round. As a result of the teaching children's knowledge improved, after the instruction some (although not all) children provided only scientifically accurate answers. The result of children's learning did not depend on their factual knowledge about the round earth. Regardless of the method of teaching, children experienced the same problems: most importantly, discord between the sensual-empirical information and that mediated through symbols (see Figure 1). Initial constraints, which are emphasised by Vosniadou (e.g. 1994a), also support the sensual-empirical knowledge.

Up / down direction. Since up/down direction is perceived as the absolute direction of a fall from a very early age (see the above) when knowledge is not yet conscious, this belief is also very difficult to change (Vosniadou 1994a). Moreover, children experience difficulties in understanding the direction of gravity because it requires a good level of visual-spatial thinking (in order to conceptualise a round earth with different directions of up/down gravity in different areas). Children explain (and, hopefully, understand) the direction of gravity better if they are taught by means of models. The model is a mediator and its application has to be learned (Grosslight \& Unger et al. 1991), which explains why children experience problems with using the model. Children have less difficulty with understanding that people walk on the earth on feet (not on their hands), but may not understand that it rains towards the earth not past the earth. For example: 
Teacher: The earth pulls people towards it, and people also live on this side of the earth [indicates downwards]. How do they live like that?

Jaan: They are living like this [puts his head against a ball]. They are living upside-down, but when they are living down below, then everything is the other way around.

Tom:There you wouldn't understand that we were living upsidedown, we would fall down.

Mikk: People on the other side walk the same way like us.

Teacher: Towards which side does it rain here? [indicates to the lower side of the ball]

Jaan: On both sides.

Teacher: It rains on the earth. Eventually, it rains on the earth. Up there it rains towards the earth, but how is it down below?

Jaan: [indicates that it rains away from the earth]

Teacher: The earth pulls everything towards it, even the rain.

Children's responses are suggestive of their synthesising information, attempting to reinterpret what they have learned, not their initial beliefs. In the following example a child uses a hollow model of the spherical earth to explain why people would not fall off the earth:

Teacher: How are people living on this spot of the earth? [points to the lower side of the ball representing the model of the earth] How can people be here - do they walk on their feet?

Heli:He could fall, but he doesn't, because it [the ball=the earth] rotates.

Anne: But in fact it is here inside the earth. Because we are here inside the earth because we couldn't walk on the earth here, we would fall off here (on the side), we are inside the earth.

Heli: No, we are still on top of the ball.

Teacher: That's right. We are on the ball, not inside. The earth pulls us towards it, otherwise we would definitely fall off.

Which objects are pulled towards the earth (i.e. which objects are affected by gravitational force)? While it has been previously mentioned that children's initial conception that in the absence of sup- 
port objects fall down, it is important to specify that their prior empirical knowledge makes it applicable only to heavy objects (e.g. people, ball, stone). Interviews revealed that some children believe that the earth does not pull objects of lighter weight. It has also been discovered that some children are on the opinion that the earth does not pull objects that are farther from the earth (e.g. in the sky, such as, for example, planes and birds). The following example illustrates the question of weight:

Teacher: Which things are affected by gravity?

Heli:People-

Anne: For example, if I jump, the earth will pull me back.

Urve: How can a bird get off the ground?

Anne: Birds are light but we are heavy, even if a baby jumped up, it would still fall down, even if a cuddly toy was thrown up in the air.

Teacher: That's right. We would always fall down. But birds have to flap their wings. As a matter of fact, gravitational force affects everything

Heli: N-no.

Teacher: Which things does it not affect then?

Heli: A plane.

The previous example demonstrates how Anne incorporates in her talk examples to illustrate her thoughts. All the conversations included here show how children attempt to combine and conceptualise information acquired from different sources.

\section{Synthetic knowledge: seasonal changes}

The reasons for seasonal changes are extremely difficult to understand, since accurate understanding requires prior knowledge of different spheres of life. On the basis of direct observation people conclude that it is the sun that moves, not the earth. Schoolchildren have almost no problems with learning the fact that it is "actually" the earth that revolves around the sun and rotates around its axis (as well as with learning the fact that the earth is round). But new questions constantly emerge - when the sun is higher up in 
the sky (i.e. appears to be further away), why is it hotter than when it is lower; how is it possible that when it is winter in some areas of the earth, it is summer in others; why is it always warmer near the equator, etc. These phenomena are usually explained through the mediation of language and models. Understanding the mechanism of seasonal changes requires knowledge of physics (of light, the travelling of light, the movement of the earth) and principles of construction and interpretation of mediators - models and figures. The necessary information is acquired in different grades and courses, and schoolchildren often fail to integrate the isolated pieces of information. This also explains why most adults fail to adequately explicate the reasons for the change of seasons (Kikas 2004; Ojala 1997).

Difficulties in understanding are best illustrated by examples from interviews from a previous study, where a group of four members was supposed to agree on why it is colder in winters and hotter in summers (Kikas 1998b). The study was conducted during the period when the topic was covered in Estonian schools during the fifth grade, and only one textbook and a book of exercises was used. The topic was also touched upon in geography classes in connection with climate and climate zones and in physics in relation to the travelling of light in older grades. 278 schoolchildren were previously tested in written form. The children were expected to shortly explain the day/night cycle and the change of seasons, and provide definitions for some terms. On the basis of the preliminary replies, 104 pupils of the third, fifth, seventh and ninth grade, with an equal number of boys and girls, were chosen to participate in the following study. Based on the preliminary test, pupils of the same grade and same gender were placed in four-member groups. The groups were formed on the principle that the individual explanations of two children in the group relied on empirical knowledge (e.g. 'Seasons change because in winters the earth is covered with snow') and the explanations of two other children relied on learned knowledge (e.g. 'Seasons change because the earth revolves around the sun').

The discussion started by answering the question 'Why is it colder in winter than in summer?' The pupils were then presented a figure of four orbits (see Fig. 3) from which they had to choose the most accurate one and justify their choice. The orbits presented 
included the one included in the textbook (a), a cross-sectional image of the orbit (b), and the orbits according to 'the distance theory' (c, d). According to the distance theory the reason why summer and winter temperatures differ lies in the varying distance between the earth and the sun - in winter the earth is farther from the sun and in summer it is closer. The theory derives from practical experience: it is warmer near the stove and cold away from it. Children and adults of different countries favour the distance theory (e.g. Ojala 1997). The schoolchildren were then asked to draw the position of the earth on the orbit in winter, spring, autumn and summer. The task objective was to arrive at a mutual agreement. The following examples are taken from the discussions of two groups of girls. Anu, Irja, Riini and Kai were ninth-graders, Malle, Pille, Ragne and Jana seventh-graders (all the names have been changed).

Figures as mediators of information. The pupils experienced serious difficulties in understanding and using figures as mediators of information. The presented orbits prompted several questions.

1.

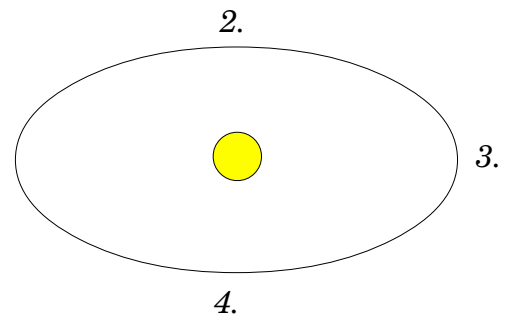

(a)

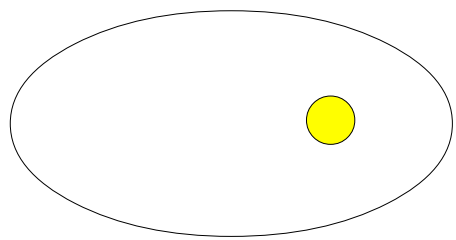

(c)

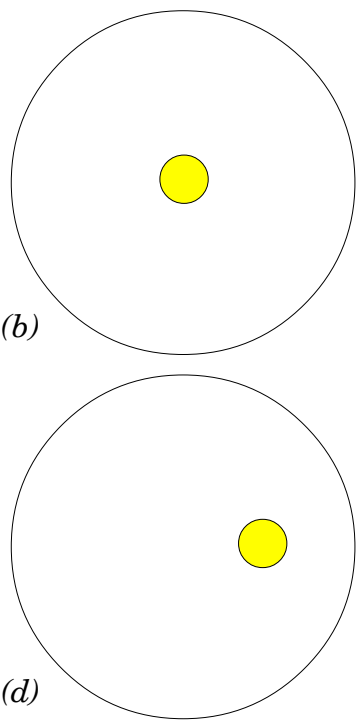

Figure 3. Figure presented to schoolchildren, depicting the sun and the four possible orbits of the earth. 
I(=interviewer): Look, here are four possible orbits of the earthwhich of these matches the actual orbit of the earth?

Anu: In summer or in general?

I: If you think it is different in summer and in winter, indicate both.

Everyone:Whoa!

I: Does any of these match? If not, draw a new one.

Anu:Well, this (a)

Irja: What, in summer?

Anu: In general.

The problems appear to arise from failure to understand the perspective from which the orbit is presented. Observing from the earth the sun seems to move differently in summer and in winter, which is why the respondents attempted to indicate different orbits for winter and for summer.

After the orbit was chosen, the respondents faced the question where the earth is located in winter and in summer. Even though according to the orbits (a) and (b) the location of the earth may principally differ, the respondents start looking for a single absolute possibility. As a result of a long discussion the group of Anu, Irja, Riini and Kai chose the orbit (a) (see Fig. 3). However, the discussion about the location of the earth on the orbit in summer and in winter proved even longer.

Anu: Here (3) it is winter.

Riin: Here (3) it is definitely spring.

Kai: No, this is not how it was in the physics textbook-

Irja: But there's really no difference-

Anu: Here (4) it is summer-

Kai: In the physics textbook it was here (1)

Anu: Let's do it as it's on the compass-

Kai: What do you mean?

Anu:Well, on the compass winter is up - the North Pole is above and the South Pole is below- 
As is evident from the example, the attempt to understand the figure was based on two sources. Firstly, previous knowledge - the north needle on the compass points up, in winter it is cold, and therefore the earth's location in winter should also be above. Secondly, the group attempted to remember how the corresponding figure was presented in the textbook. Many groups indicated to the textbook orbit and therefore chose the orbit (a), although they failed to justify their choice. The following discussion illustrates the encountered difficulties:

Pille: I think it should be that one (a).

Malle: Why, why should it be so irregular?

Pille: Because this is how it was on the picture, because this is how it was on the picture.

Malle: Why would it go further away from the sun... if it already has an orbit why would it go further away?

Pille: I don't know, I've always had the impression that it is not altogether round, its journey-

Ragne: I think so, too (a)-

Jana: I think, too (a)

Malle:Well, I still think that if they already have certain orbits then their position relative to the sun should always be the same.

Pille: I don't know how I know, I don't know why, it is everyhere that it is not totally round, I've even read it someplace that it is not totally round, everywhere where- it is shown what is it like, it is never totally round, it has always been somewhat oval Malle: Well, how could it be sometimes further away and sometimes closer?

Ragne: I don't know-

Malle:Your reasoning is based on that you've seen it someplace, but why can't I see it like that then?

Pille: I'm not exactly sure, but everywhere where it is shown it has never been totally round.

Malle:Wait a minute! The sun is virtually round, isn't it. So let's suppose that the sun has some kind of gravitationalforce... be- 
cause they move the same way all the time, that is why it is on the orbit, otherwise it may go randomly wherever... But why should it sometimes go further, is one side of the sun different than the other...?

Malle: Is the sun then oblate?

Pille: Well, how do you know that the sun is round?

Malle:I think it is, when the planets and things are all round. Pille: Well, they are not exactly round.

Malle:Not exactly, but almost round ... the sun is the same all over, I think that the sun is round.

This particular group could not arrive at an agreement, as Malle chose the round orbit (b) and the others decided upon the ellipse (a), which clearly manifests the impact of textbook knowledge - the figure does not have to be comprehensible but as long as it is included in a textbook, it must be the only correct possibility. Malle's logical reasoning (the sun is round, the sun's gravity is the same on all sides) did not convince others, even though they had no other arguments in favour of the elliptical orbit.

Distance theory and problems connected with its association. Distance theory was the most accessible explanation, as many groups proceeded from it. Problems arose only when the respondents were expected to explain how the temperature of different areas of the earth varies (e.g. in terms of orbits (c) or (d)), or how is it possible that the earth is located at the same distance in both winter and summer (orbits (a) or (b)). Children recall the acquired knowledge, provide examples and analogies from other areas by attempting to construct coherent explanations. The group of Anu, Irja, Riini and Kai offered the following explanations:

Kai: These eastern winds are cold winds, while the western winds are warmer, milder winds, and, actually, southern winds are warmer, and perhaps it is the same on the sun that it warms one side more, in summer as if comes from the west and then the air is warmer, but in winter comes from the east and the air is colder (orbit a)

I: So it warms differently? 
Kai:Yes-

I: But how do you explain that when it is summer here [in Estonia], it is winter in Australia.

Kai: But when the sun shines from the west for us, it also...shines from the west for them.

Irja: Are there any planets inside?

Kai: Yes, there are two.

Irja: But these might get in the way here-

Anu:Yes, and the sun cannot shine on the third planet, if the others are in the way, and here, on the other side, where it is summer, the planets have moved on and won't get in the way at all.

I: That's a nice idea, but how do you explain that when it is summer here, it is winter in Australia?

Anu:But...the northern side remains to the side of the sun and the southern side is away from the sun.

Everybody: Yes, Estonia is here and Australia is over here.

Anu: And the sun shines on Estonia and doesn't shine on thatEverybody: Yes, exactly.

This example shows that the pupils attempt to construct explanations by using and synthesising information from various spheres of life (cf. Brewer \& Chinn et al. 2000). They make a reference to their own experience, to what they have read and seen in a textbook, recall facts, analogies with other phenomena, make logical conclusions. And still they arrive at explanations which in specific literature are called misconceptions or distorted knowledge (Brewer \& Chinn et al. 2000; Driver \& Squirer et al 1995). Problems are encountered while trying to incorporate different pieces of information and arranging the information acquired through different mediators.

\section{CONCLUSION}

There is an array of material and mental mediators which can be used to construct explanations about the world. Different media- 
tors are used for creating different explanations (models, theories); difficulties in understanding knowledge may arise from the fragmentariness of the acquired information, and the pieces of information may prove inconsistent. Additional problems may emerge when the characteristic features of mediators and mediation are misunderstood. Children experience problems in understanding phenomena as well as in imparting knowledge, because they are in the middle of the process of learning to use different mediators.

In reality, various systems of concepts and knowledge are available for adults. By starting to use new (material and mental) mediators, the old ones are still available for them (e.g. Chi 1992; Mortimer 1995). While communicating with children or attempting to understand old theories, people may encounter problems with only onealthough the most modern - type of mediators and knowledge. Everyday life requires not only academic reasoning but also common thinking. Even though Einstein's theory is more comprehensive and generalised, it is still the Newton's law that is generally applied.

Problems in conceptualising and expressing knowledge are clearly evident in constructing explanations about natural phenomena caused by the earth, the sun and planets, and their movement. Reasoning and thinking within the frameworks of different systems was evident in previous examples, where arguments from everyday experience and formal education were incorporated in the discussion. Children construct explanations based on knowledge acquired through practical experience and in school, making conclusions according to their level of development. The explanations and justifications of children and adults may indeed be different, but both strive for congruity which would lead to the sense of understanding.

\section{Acknowledgements}

The collection of the material and the preparation for the article has been supported by the Estonian Science Foundation (grant no. 5371). Children were instructed by Hele Kanter and Triin Hannust, who also collected a part of data. All remaining errors are my own and not theirs. 


\section{References}

Baillargeon, Rene 1995. Physical reasoning in infancy. The Cognitive Neurosciences. Gazzaniga, Michael (ed.). Cambridge, MA: MIT Press, pp. 181-204.

Blades, Mark \& Spencer, Christopher 1994. The development of children's ability to use spatial representations. Advances in Child Development, 25. Reese, Hayne (ed.). San Diego: Academic press, pp. 157199.

Brewer, William \& Chinn, Clark \& Samarapungavan, Ala 2000. Explanation in scientists and children. Explanation and Cognition. Keil, Frank \& Wilson, Robert (eds.). Cambridge, MA: MIT Press, pp. 279-298.

Callanan, Maureen \& Oakes, Lisa 1992. Preschoolers' questions and parents' explanations: Causal thinking in everyday activity. Cognitive Development, 7, pp. 213-233.

Carey, Susan 1985. Conceptual Change in Childhood. Cambridge, MA: MIT Press.

Chi, Michelene 1992. Conceptual change within and across ontological categories: Examples from learning and discovery in science. Giere, R. (ed.). Cognitive models of science. Minnesota Studies in Philosophy of Science, 15. Minneapolis: University of Minnesota Press, pp. 129-187.

Chinn, Clark \& Brewer, William 2000. Knowledge change in response to data in science, religion and magic. Rosengren, Karl \& Johnson, Carl $\&$ Harris, Paul (eds.). Imagining the Impossible. Magical, Scientific, and Religious Thinking in Children. New York: Cambridge University Press, pp. 334-371.

Diakodoy, Irene-Anna \& Vosniadou, Stella \& Hawks, Jackson 1997. Conceptual change in astronomy: models of the earth and of the day/ night cycle in American-Indian children. European Journal of Psychology of Education, 12, pp. 159-184.

Driver, Rosalind \& Squires, Ann \& Rushworth, Peter \& Wood-Robinson, Valerie 1995. Making Sense of Secondary Science: Research into Science Ideas. London \& New York: Routledge.

Glynn, Shawn \& Duit, Rene (comp.) 1995. Learning Science in the Schools. Mahwah, NJ: Erlbaum.

Gopnik, Alison \& Meltzoff, Andrew 1997. Words, Thoughts, and Theories. Cambridge, MA: MIT Press.

Grosslight, Lorraine \& Unger, Christopher \& Jay, Eileen \& Smith, Carol 1991. Understanding models and their use in science. Journal of Research in Science Teaching, 28, pp. 799-822.

Hannust, Triin \& Kikas, Eve 2002. Five- and seven-year-old children's concepts of the earth and the influence of experimental teaching on these concepts. A Process Approach to Conceptual Change. Proceedings of the Third European Symposium on Conceptual Change, June 26-28, Turku, Finland, pp. 176-183. 
Karmiloff-Smith, Anette 1992. Beyond Modularity: A Developmental Perspective on Cognitive Science. Cambridge, MA: MIT Press.

Kikas, Eve 1998a. The impact of teaching on students' definitions and explanations of astronomical phenomena. Learning and Instruction, 8, pp. 439-454.

Kikas, Eve 1998b. Pupils' explanations of seasonal changes: age differences and the influence of teaching. British Journal of Educational Psychology, 68, pp. 505-516.

Kikas, Eve 2000. The influence of teaching on students' explanations and illustrations of the day/night cycle and seasonal changes. European Journal of Psychology of Education, 15, pp. 281-295.

Kikas, Eve 2003. Constructing knowledge beyond senses: worlds too big and small to see. Toomela, Aaro (ed.). Cultural Guidance in the Development of the Human Mind. Westport, CT \& London: Ablex, pp. 211-227.

Kikas, Eve 2004. Teachers' conceptions and misconceptions concerning three natural phenomena. Journal of Research in Science Teaching, 5, pp. 432-448.

Kikas, Eve \& Hannust, Triin, \& Kanter, Hele 2002. The influence of experimental teaching on 5- and 7-year-old children's concepts of the earth and gravity. Journal of Baltic Science Eduation, 2, pp. 19-30.

Kuhn, Thomas 1962. The Structure of Scientific Revolutions. Chicago: University of Chicago Press.

Kuperjanov, Andres 2003. Eesti taevas. Uskumusi ja tõlgendusi [The Estonian Sky. Beliefs and Interpretations]. Tartu: Eesti Folkloori Instituut.

Mortimer, Eduardo Fleury 1995. Conceptual change or conceptual profile change? Science and Education, 4, pp. 267-285.

Nelson, Katherine 2003. Making sense in the world of symbols. Toomela, Aaro (ed.). Cultural Guidance in the Development of the Human Mind. Westport, Connecticut \& London: Ablex, pp. 139-158.

Nobes, Gavin \& Moore, Derek \& Martin, Alan \& Clifford, Brian \& Butterworth, George \& Panagiotaki, Georgia \& Siegal, Michael 2003. Children's understanding of the earth in a multicultural community: mental models or fragments of knowledge? Developmental Science, 6, pp. 74-87.

Ojala, Jorma 1997. Lost in space? The concepts of planetary phenomena held by trainee primary school teachers. International Research in Geographical and Environmental Education, 6, pp. 183203.

Samarapungavan, Ala \& Vosniadou, Stella \& Brewer, William 1996. Mental models of the earth, sun, and moon: Indian children's cosmologies. Cognitive Development, 11, pp. 491-521.

Schoultz, Jan \& Säljö, Roger \& Wyndhamn, Jan 2001. Heavenly talk; Discourse, artifacts, and children's understanding of elementary astronomy. Human Development, 44, pp. 103-118. 
Siegler, Robert 1996. Emerging Minds. The Process of Change in Children's Thinking. New York \& Oxford: Oxford University Press.

Spelke, Elizabeth 1991. Physical knowledge in infancy: reflections on Piaget's theory. Carey, Susan \& Gelman, Rochel (eds.). The Epigenesis of Mind: Essays on Biology and Cognition. Hillsdale, NJ: Erlbaum, pp. 133169.

Tomasello, Michael 2000. The Cultural Origins of Human Cognition. Cambridge, MA \& London: Harvard University Press.

Toomela, Aaro 2003. Culture as a semiosphere: On the role of culture in the culture-individual relationship. Josephs, Ingrid (ed.). Dialogicality in development. Westport, CT: Praeger, pp. 129-163.

Vosniadou, Stella 1994a. Capturing and modeling the process of conceptual change. Learning and Instruction, 4, pp. 45-69.

Vosniadou, Stella 1994b. Universal and culture-specific properties of children's mental models of the earth. Hirschfeld, Lawrence \& Gelman, Susan (eds.). Mapping the Mind: Domain Specificity in Cognition and Culture. Cambridge, MA: Cambridge University Press, pp. 412-429.

Vosniadou, Stella \& Brewer, William 1992. Mental models of the earth: A study of conceptual change in childhood. Cognitive Psychology, 24, pp. 535-585.

Vosniadou, Stella \& Brewer, William 1994. Mental models of the day/ night cycle. Cognitive Science, 18, pp. 123-183.

Vygotsky, Lev 1934/1997. Thought and language. Revised edition of 1986. Cambridge, MA: MIT Press.

Vygotsky, Lev 1931/1983. Istoriia razvitiia vyschikh psikhicheskikh funktsii [History of the Development of Higher Psychical Functions]. Sobranie sochinenii, 3. Moscow: Pedagogika, pp. 5-228.

Vygotsky, Lev \& Luria, Alexander 1994. Tool and symbol in child development. The Vygotsky Reader. Van der Veer, Rene \& Valsiner, Jaan (eds.). Oxford \& Cambridge, UK: Blackwell, pp. 99-175.

Wellman, Henry \& Hickling, Anne \& Schult, Carolin 1997. Young children's psychological, physical, and biological explanations. Wellman, Henry and Inagaki, Kayoto (eds.). Emergence of Core Domains of Thought: Children's Reasoning about Physical, Psychological, and Biological Phenomena. San Francisco, CA: Jossey-Bass, pp. 7-25.

Wertsch, James 1998. Mind as Action. New York \& Oxford: Oxford University Press.

Wertsch, James 1985. Vygotsky and the Social Formation of Mind. Cambridge, MA: Harvard University Press. 